\title{
Wireless Communication with Smart Photonic Antennas using Transmission Power Control
}

\author{
Georgios Tzeremes, Herbert Tanner, Tsai Liao and Christos Christodoulou
}

\begin{abstract}
This paper presents a novel, integrated a photonic antenna with a power control scheme. The controller regulates the levels of radiated power by adjusting the biasing voltage of a side-illuminated waveguide photodetector (WGPD). The WGPD converts the RF-modulated optical power into a microwave signal, which is then fed to an antenna. The controller uses as input the noise level measurements of a different receiving antenna, and periodically calculates the necessary amount of power that must be radiated. The performance of the RF/photonic device has been studied theoretically and in simulation. With the addition of power control capabilities, the device realizes an early attempt to design smart photonic antennas.
\end{abstract}

\section{INTRODUCTION}

There are three main steps in the evolution of $\mathrm{RF} /$ Photonics systems for wireless communications. The first step is to use photonics to gradually replace conventional RF components, such as the coax interconnecting the antenna to the electronics since optical fibers provide a better medium for broadband RF communication systems. The light weight property of fibers, and its immunity to other signal interference make them ideal in the development of future RF distribution systems. The second step is the seamless integration of photonics and RF wireless circuits in the form of OptoElectronic Integrated Circuits (OEIC's); fiber-optic technologies have reached the stage where insertions into various commercial RF systems are practical. In the third step the aim is to eliminate the need of local oscillators, mixers, amplifiers and a host of other parts by directly feeding an antenna through a fiber at millimeter wave frequencies. Despite the fact that the integration of photonics with antennas has recently gained momentum [2], [3], [4], the issue of controlling the output power in photonic antennas has not yet been considered.

We present a framework for developing faster, adaptive wireless communication using arrays of smart antennas. Our primary contribution is on the integration of RF antennas with optical fibers and transmission power control systems. The antenna and the optical fiber are interfaced through a photodetector, and the hybrid integration with the antenna increases bandwidth and communication capacity. Our power control system (implemented in software) uses RF noise power measurements to adaptively regulate the bias voltage of the photodetector. Through the bias voltage, the transmission power of the antenna can

Georgios Tzeremes and Christos Christodoulou are with the Department of Electrical and Computer Engineering of the University of New Mexico, Albuquerque, NM 87131 \{gtzerem, cgc \} @eece. unm.edu.

Herbert Tanner is with the Department of Mechanical Engineering of the University of New Mexico, MSC01 1150, Albuquerque, NM 87131, tanner@me.unm.edu.

Tsai Liao is with the Department of Electrical Engineering, University of California, San Diego. be adjusted. In this way we achieve the required signal-tonoise ratio for slowly varying noise levels, and control the capacity of the channel at the same time.

Since parallel nodes in a wireless network introduce noise, nodes must transmit at the minimum possible energy level. This paper presents a method to automatically regulate the transmission power of each antenna so that the capacity of the channel is adjusted, and signal interference is kept at minimum. An array of RF modulator/photodetectors integrated directly to an array of antennas, can combine the advantages of both fiber optics and wireless channels. Used with a transmission power control system, this new $\mathrm{RF}$ /photonic antenna array can form a smart system that enhances network capacity and coverage. A large number of such $\mathrm{RF} /$ Photonic antenna elements could be networked together into a star configuration, feeding in and out of a radio hub.

\section{The Photodetector-Antenna System}

The flexibility in the design of Waveguide Photodetector (WGPD) - provided by optical coupling, optical absorption, transit time and capacitance - offers the ability to optimize it for the given application [5], [6]. The Waveguide Photodetector converts the RF-modulated optical power into a microwave signal, subsequently fed to an antenna. Between the WGPD and the antenna there is a double stub matching circuit, to ensure the maximum transfer of power from the WGPD to the radiating elements. The general concept of this design from the optical modulation, to the optical fiber and finally the radiating elements is presented in Figure 1. The WGPD is a standard p-i-n device grown on a semi-insulated InP substrate. It eliminates the bandwidth-efficiency trade-off that is fundamental to normal, surface illuminated photodetectors.

The photodetector is fed by an optical fiber, terminated at the facet of the optical waveguide layer. Bias across the photodetector affects simultaneously the output power and the frequency of operation. Increasing the biasing voltage, raises the maximum output power and decreases the pulse duration. The effects of biasing can be seen in Figure 2: biasing of the photodetector increases the drifting component in the current density equations [7]:

$$
\begin{aligned}
J_{n} & =\overbrace{q \mu_{n} n \mathcal{E}}^{\text {drift }}+\overbrace{q D_{n} \nabla n}^{\text {diffusion }} \\
J_{p} & =q \mu_{p} p \mathcal{E}+q D_{p} \nabla p \\
J_{\text {cont }} & =J_{n}+J_{p} .
\end{aligned}
$$

The biasing of the photodetector is provided through the antenna design. The antenna is a CPW- fed three element 


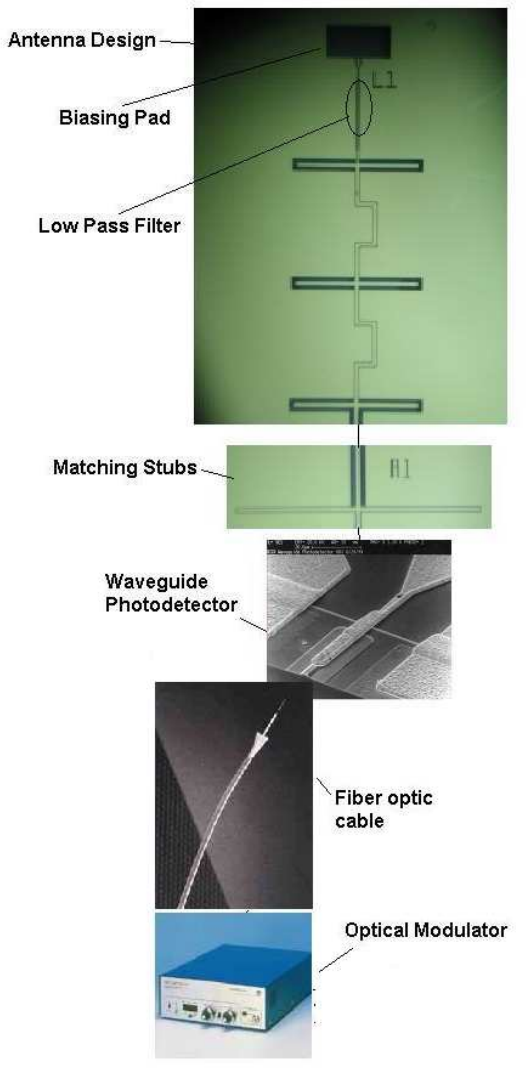

Fig. 1. Schematic representation of the antenna/photodetector.

folded dipole slot. The transmitting antenna is a transformation of the basic folded dipole design in a patch/slot version [8]. It operates around $18.5 \mathrm{GHz}$ with a bandwidth of $1 \mathrm{GHz}$ and a gain of $6.5 \mathrm{~dB}$. Due to magnetic currents generated in the slots, each folded slot antenna radiates like a half wavelength folded dipole with linear polarization. The main advantages are low cross polarization $(\leq$ $-23 \mathrm{~dB}$ 's), and low coupling between its elements [9].

To avoid leakage of the generated microwave, low pass filters have been designed for the Co-planar Waveguide (CPW) transmission line [10] (Figure 1). The CPW is used as a feed to the antenna while also serving as electrodes and biasing line of the WGPD [11]. The characteristic impedance and phase velocity of a CPW depends more on the dimensions of the conductive surface than the substrate thickness. Bending the transmission line ensures good radiation pattern in the dielectric. At the same time, this configuration preserves the length of the feeding line [12]. Figures 3 and 4 show experimental S11 parameter and the radiation pattern of the specific design.

A second antenna is used as a receiver, to monitor the background noise (Figure 5). It is a single element folded dipole patch antenna with polarization perpendicular to the transmitting antenna. The separation distance of the two antennas is over $1 \mathrm{~cm}$ (approximately 4 wavelengths), which is sufficient to avoid any coupling effects [1]. This antenna should have almost the same efficiency as the transmitting antenna, although its directivity is much smaller

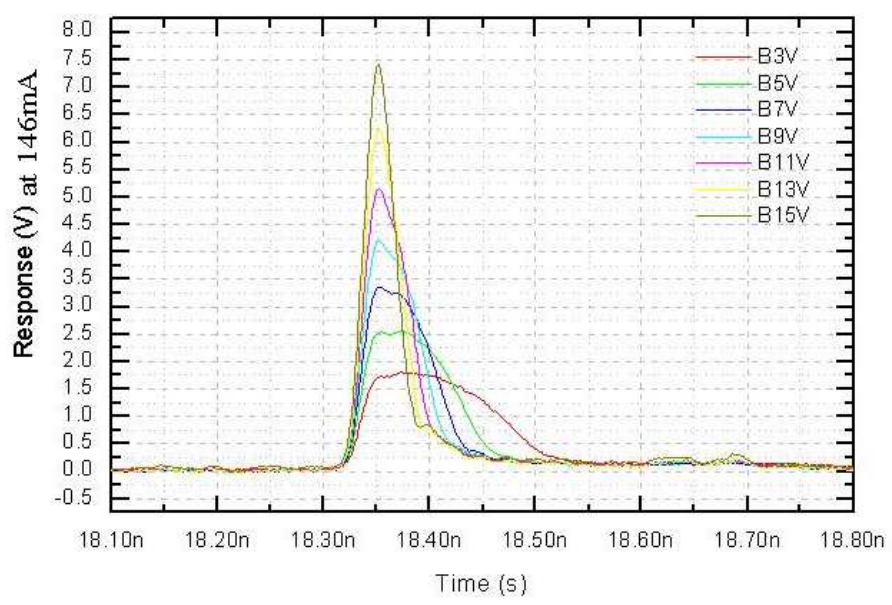

Fig. 2. Effect of PD bias voltage on transmission power.

(around $3.2 \mathrm{~dB}$ ). This provides measurements of noise from every direction in the environment, while it blocks the polarization state of the radiated signal.

\section{The Transmission Power Control System}

The objective of the control system is to use the noise measurements obtained by the second antenna in order to regulate the $\mathrm{RF}$ power levels so that, despite variations in the noise levels, the required signal-to-noise ratio is always achieved. In a large scale wireless network, such control laws have to be implemented in distributed and decentralized fashion. Figure 6 shows how this control system is interfaced with the Photodetector/Antenna: light coming from the optical fiber is transformed into RF by the Photodetector/Antenna. The bias voltage of the photodetector behaves as the controlling gate, regulating the output power level of the antenna, $P$ as $P=E v$, where $E$ is a constant that is obtained from Figure 2, and is estimated to be approximately $E=75.5 \mathrm{~mW} / \mathrm{V}$.

Due to the transmission of other antennas and reflections of its own signal, the antenna transmits in an environment with noise, $w$. The noise signal, $w$ is considered as white Gaussian. In an indoor environment, the noise signal is slowly varying, in which case the bandwidth of the power control system is usually adequate for tracking noise levels. Noise levels are picked up by a second antenna, perpendicularly polarized, in a distance sufficient to exclude coupling effects. This second antenna works as a sensor, measuring only the environmental noise: $P^{\prime}=w$. The bias voltage, $v$, is controlled by a microprocessor, based on the sequence of light pulses carrying microwave-optical signals that arrive through the optical fiber and the noise measurements obtained from the secondary antenna. The microprocessor directly regulates the bias voltage, and therefore the discrete time dynamics of the latter can be expressed as:

$$
v_{k+1}=v_{k}+u_{k}+\delta,
$$

where $u_{k}$ is the control input for power regulation and $\delta$ is frequently appearing slowly varying DC signal, acting as an input disturbance. In our setup, $\delta$ could be in the order 


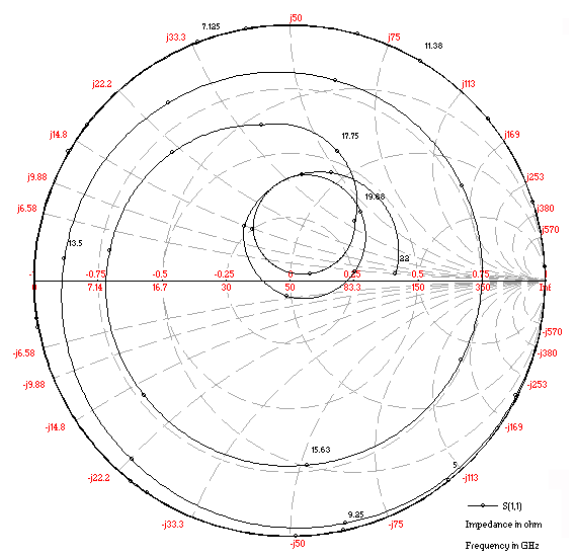

Fig. 3. S11 parameters of the transmitting antenna.

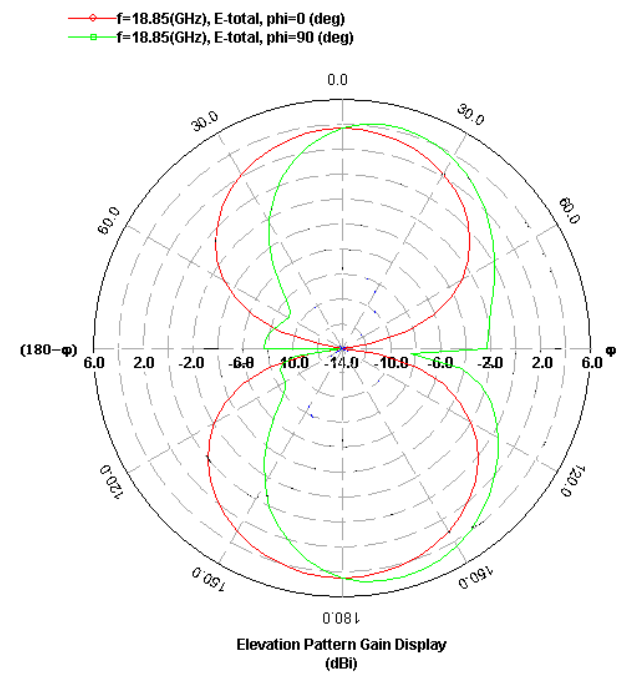

Fig. 4. The radiation pattern of the transmitting antenna.

of millivolts, whereas the bias voltage $v$ is in the order of tens of volts, allowing us to safely ignore its effect. The system output signal (Figure 8 ) is defined as:

$$
y_{k}=P-w=E v_{k}-w,
$$

and expresses the difference between the power of the transmission signal and the noise level. For successful transmission it is necessary that this difference is always above a threshold, i.e. the desired signal-to-noise ratio: $y_{\text {desired }}=\mathrm{SNR}$. The power control law can then be defined as (Figure 8):

$$
u_{k}=\frac{1}{E}\left(r_{k} \cdot \mathrm{SNR}-y_{k}\right)=\frac{r_{k} \cdot \mathrm{SNR}-E v_{k}+w}{E},
$$

where $r_{k}$ is the (light) reference signal. The simplicity of the control input (2) ensures minimum computation delay. Thus the frequency of the control loop is essentially determined by the availability of sensing data from the monitoring antenna. Bearing in mind that the system directly measures noise $w$, rather than output signal $y_{k}$, the

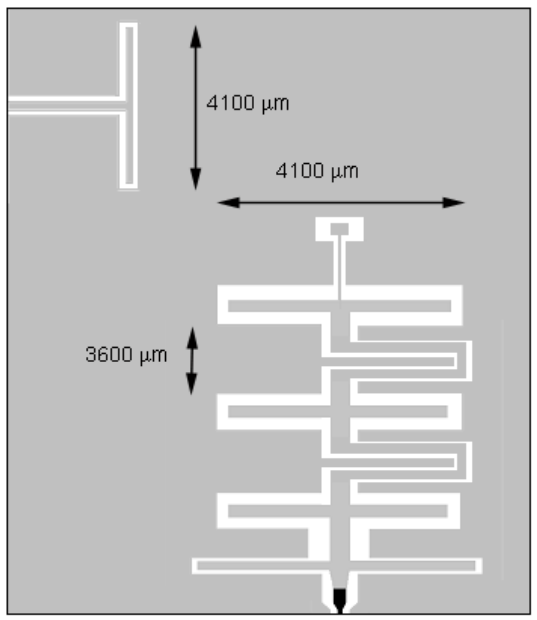

Fig. 5. Layout of the transmitting antenna and the perpendicular polarized antenna monitoring noise.

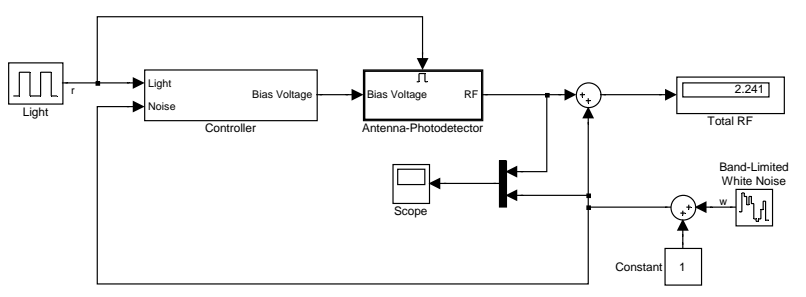

Fig. 6. Block diagram of the RF power transmission control system.

controller structure depicted in Figure 8 has to be implemented as shown in Figure 9.

The control system described above allows the photodetector/antenna system to track the noise levels and maintain a constant power level above them (Figure 10, 11).

In Figure 10, the high frequency pulse sequence is the response of the Photodetector/Antenna system to the light input signal. The low frequency pulse sequence corresponds to piecewise constant, white Gaussian noise. Regardless of the variation of the noise levels, the output is regulated to a predetermined level above that of noise.

To test the tracking performance of the control scheme further, we simulated a sinusoidal noise waveform. The results are given in Figure 11. From the response, it is clear that tracking ability is not restricted to the particular class of piecewise constant inputs but it extends to more general dynamic signals.

\section{Conclusions}

We present a novel smart antenna design, in which we integrate photodetectors with antennas and regulate the power level of radio transmission through a softwareimplemented control system. Linking the optical fiber directly with the antenna through a photodetector increases bandwidth without the need for signal amplification. The control system then adjusts the transmission power so 


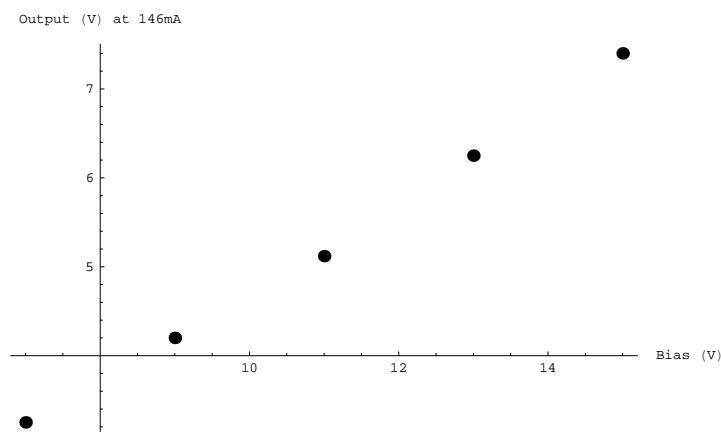

Fig. 7. Linear increase of transmission power with bias voltage.

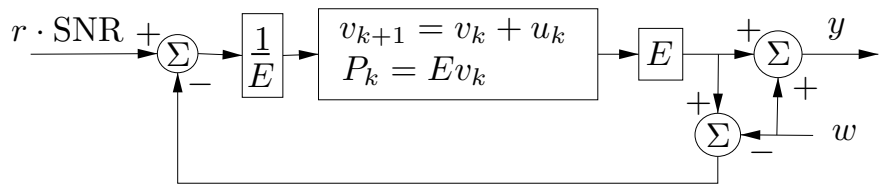

Fig. 8. Block diagram of the control system design.

that the desired signal-to-noise ratio is achieved and maintained, regardless of (low frequency) fluctuations of noise levels, ensuring reliable communication and allowing regulation of the channel capacity. The design would enable control of faster, more reliable and adaptive wireless communication systems using integration of photonics and microwave technologies.

Acknowledgement. The authors wish to thank Sayan D. Mukherjee for constructive suggestions and acknowledge partial support by NSF.

\section{REFERENCES}

[1] G.P. Gauthier, J.-P. Raskin and G.M. Rebeiz, "A 140-170$\mathrm{GHz}$ low-noise uniplanar subharmonic Schottky receiver" IEEE Transactions on Microwave Theory and Techniques, Vol. 48, No. 8 , pp. 1416-1419, August 2000.

[2] A. Hirata, T. Nagatsuma, " $120 \mathrm{GHz}$ millimeter-wave antenna for integrated photonics transmitter," Electronics Letters, Vol. 34, No.24, pp. 1460-1461, November 2001.

[3] Siou Teck Chew, Tak Kit Tong, Ming C. Wu, and Tatsuo Itoh, "An Active Phased Array with Optical Input and Beam-Scanning Capacity," IEEE Microwave and Guided Wave Letters, Vol. 4, No. 10, pp. 347-379, October 1994.

[4] Majid Khodier, George Tzeremes, Tsai S. Liao, Paul K. L. Yu and C. G. Christodoulou, "Smart RF/ Photonic Antennas' for Ultra high capacity Wireless Communications," Proceedings SPIE Digital Wireless Communications IV, June 2002, Vol. 4740, pp. $132-141$.

[5] K. Kato, S. Hata, K. Kawano, J. Yoshida and A. Kozen, "A High Efficiency $50 \mathrm{GHz}$ InGaAs Multimode Waveguide Photodetector," IEEE Journal of Quantum Electronics, Vol. 28, pp. 2728-2735, December 1992.

[6] H. Jiang and P. K. L. Yu, "High power waveguide integrated photodiode with distributed absorption," IEEE MTT-S Symposium Digest, Vol. 2, pp. 679-682, 2000.

[7] S. M. Sze, Physics of Semiconductor Devices, Second Edition, John Wiley \& Sons, 1981.

[8] George Tzeremes, Tsai Liao, Paul K. Yu, Christos Christodoulou, "Computation of equivalent circuit models of optically driven CPW-fed slot antennas for wireless communications," IEEE Antennas and Wireless Propagation Letters, Vol. 2, No. 10, pp 140-142, 2003.

[9] George Tzeremes, Paul K. Yu, Christos Christodoulou, "Integration Issues of a Waveguide Photodetector with a CPW fed

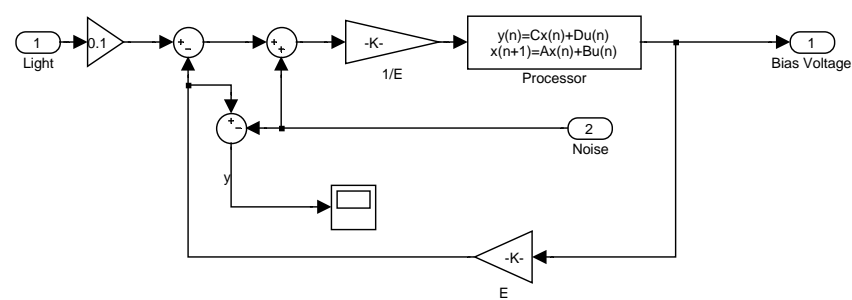

Fig. 9. Matlab Simulink implementation of the control system.

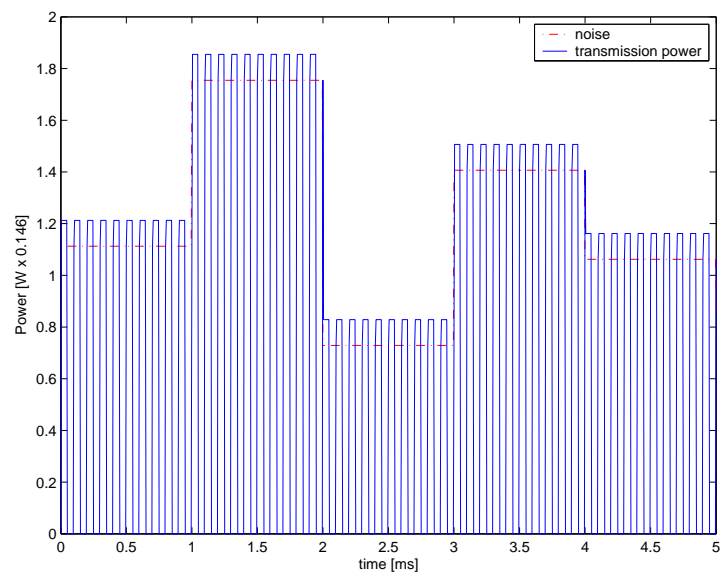

Fig. 10. Tracking piecewise constant (white) noise levels.

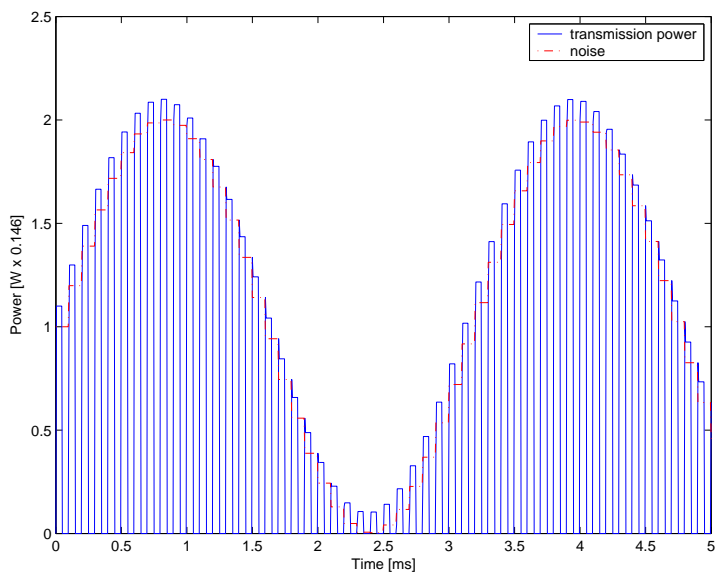

Fig. 11. Tracking sinusoidal reference noise signals.

three element slot antenna," Proceedings of the International Symposium of the Antennas and Propagation Society, Monterey, 2004.

[10] K. C. Gupta, R. Garg, and I. J. Bahl, Microstrip Lines and Slotlines, Artech House, 1979.

[11] H. G. Rao, Q. Z. Liu, A. L. Kellner, X. S. Jiang, S. S. Lau and P. K. L. Yu, "Microwave characteristics of Coplanar waveguides on helium implanted epitaxial p-InP," Applied Physics Letters, Vol. 66, pp. 1933-1935, 1995.

[12] Tomas M. Weller, Linda P. B. Katehi, Gabriel M. Rebeiz, "Single and Double Folded Slot Antennas on Semi Infinite Substrates," IEEE Transactions on Antennas and Propagation, Vol 43, No. 12, December 1995. 